\title{
The Association of Prevalent Kidney Stone Disease with Mortality in US Adults: The National Health and Nutrition Examination Survey III, 1988-1994
}

\author{
Jie Tang ${ }^{a}$ Pam Mettler $^{b} \quad$ Kim McFann $^{b} \quad$ Michel Chonchol ${ }^{a}$ \\ a Division of Renal Disease and Hypertension, University of Colorado School of Medicine, Aurora, Colo., and \\ ${ }^{b}$ Colorado School of Public Health, University of Colorado, Denver, Colo., USA
}

\section{Key Words}

Mortality · Cardiovascular disease · Kidney stone disease

\begin{abstract}
Background: Kidney stone disease is associated with hypertension, diabetes, metabolic syndrome, kidney function decline, and increased cardiovascular (CV) events. However, its association with all-cause and CV mortality is unclear. Methods: We used the Third National Health and Nutrition Examination Survey, a large US population-based study with mortality data through 2006 determined via linkage to the National Death Index to examine kidney stone disease in relation to all-cause and CV mortality risks. Results: Among 14,879 men and women over 18 years of age who were eligible for analysis, 683 participants reported a history of kidney stones. There was a total of 3,590 all-cause and 1,608 CV deaths during a median follow-up of 14.9 years. Stone formers had a significantly higher risk for allcause mortality [hazard ratio (HR): $1.95,95 \% \mathrm{Cl}: 1.64-2.33$, $\mathrm{p}<0.0001$ ) and CV mortality (HR: $2.05,95 \% \mathrm{Cl}: 1.60-2.62$, $\mathrm{p}<0.0001)$ in unadjusted analyses. However, after multivariate adjustment for age, gender, race, and poverty, stone formers no longer had increased risk for all-cause mortality (HR: 1.08, 95\% Cl: 0.93-1.26, $\mathrm{p}=0.3$ ) and CV mortality (HR: $1.07,95 \% \mathrm{Cl}: 0.84-1.36, \mathrm{p}=0.6)$. Results remain unchanged after further adjustment for other clinical vari-
\end{abstract}

ables including history of hypertension, diabetes, and CV disease. Conclusion: The increased risk of all-cause and CV mortality in kidney stone formers is likely a reflection of unique demographics and associated comorbidities. There is no independent association of prevalent kidney stone disease with all-cause and CV mortality.

Copyright $\odot 2013$ S. Karger AG, Basel

\section{Introduction}

Kidney stone disease is common in industrialized countries, affecting $10-15 \%$ of males and $3-5 \%$ of females in the United States [1], and its prevalence is still rising [2]. It causes significant morbidities and has a vast economic impact [3].

Kidney stone disease has been increasingly recognized as a complex systemic illness. Studies have linked kidney stone disease to a variety of cardiovascular $(\mathrm{CV})$ risk factors including hypertension, diabetes, and metabolic syndrome $[4,5]$. More recently, Reiner et al. [6] analyzed data among participants in the CARDIA study, and demonstrated that young adults with a history of kidney stone had a higher prevalence of subclinical atherosclerosis. Similarly, Rule et al. [7] also showed that stone formers had a significantly higher risk for myocardial infarction. Furthermore, kidney stone disease has also been

\section{KARGER}

E-Mail karger@karger.com

www.karger.com/ajn
C 2013 S. Karger AG, Basel

0250-8095/13/0375-0501\$38.00/0
Jie Tang, MD

Division of Renal Disease and Hypertension

University of Colorado School of Medicine

Aurora, CO 80045 (USA)

E-Mail Jie.tang@ucdenver.edu 
associated with an increased risk of kidney function decline and incident end-stage renal disease $[8,9]$.

Despite these strong associations between kidney stone disease and other systemic illnesses that are important and well-established predictors of survival, no studies to date have examined the independent relationship between allcause and $\mathrm{CV}$ mortality risk and kidney stone disease. Therefore, in this study we aim to examine the relationship between kidney stone disease and all-cause and CV mortality among the US adult population using the Third National Health and Nutrition Examination Survey, 1988-1994 (NHANES III).

\section{Subjects and Methods}

\section{Study Population}

NHANES III is a national probability sample of the total noninstitutionalized civilian population, 2 months of age or over in the United States. It is a cross-sectional study and was conducted from 1988 to 1994 . The survey collected demographic, socioeconomic, dietary, and health-related information, in addition to the examination and laboratory data obtained by highly trained medical personnel.

There were a total of 33,994 participants in NHANES III, and our analyses were limited to 20,024 adult participants 18 years or older who had mortality data. Among those, 19,597 responded 'yes' or 'no' to the question regarding the history of kidney stones. Responders who did not have complete information on physical activity or poverty-income ratio (PIR); had missing body mass index (BMI); did not have measurements of serum C-reactive protein (CRP), total cholesterol (TC), high-density lipoprotein (HDL), and serum 25 -hydroxy vitamin $\mathrm{D}(25(\mathrm{OH}) \mathrm{D})$; were missing estimated glomerular filtration rate (eGFR) as measured by the Modification of Diet in Renal Disease (MDRD) equation, or had incomplete data for the history of hypertension, diabetes, and CV disease were excluded $(n=4,718)$. Thus, the final sample used in this study included 14,879 adult participants.

\section{Primary Predictor and Outcome}

The primary predictor or independent variable was prevalent kidney stone disease. It was extracted from the interview data file. 'Have you ever had a kidney stone?' was the question asked during the standardized home interview. Adult participants who responded 'yes' to the question were considered to have a history of kidney stone in life. Participants were also asked the number of different occasions they passed a kidney stone.

The outcome or dependent variable of interest was all-cause and CV mortality. To evaluate mortality we used the NHANES III linked mortality file. This uses probabilistic record matching with the National Death Index through December 31, 2006 to determine the mortality status among NHANES III eligible participants. International Classification of Diseases, Tenth Revision (ICD10) codes were used to identify participants for all-cause mortality, as well as for whom CV disease was listed as the underlying cause of death (ICD10 I00-I78). A detailed description of matching methodology is available [10].

\section{Covariates}

Age was defined as age at the time of the interview. Race/ethnicity was self-reported as non-Hispanic white (NHW), non-Hispanic black (NHB), Mexican American (MA), or other. A history of hypertension was defined as a self-reported physician diagnosis of hypertension, ever having been asked to take blood pressure medication, or taking blood pressure medication at the time of interview. A history of diabetes was defined as a self-reported physician diagnosis of diabetes or taking insulin at the time of interview. A history of CV disease was defined as having at least one of the following: self-reported physician diagnoses of acute myocardial infarction, stroke, or heart failure. Physical activity assessment was part of the comprehensive interview. Participants were asked to identify various exercises during their spare time. They were asked to specify the number of times they engaged in an identified activity during the past month. The frequency of other exercises, sports, or activities was also recorded. The physical activity was specified as the sum of intensity rating multiplied by times (of each activity) every month. PIR was obtained by dividing family income by the poverty threshold and was used as the indicator of socioeconomic status. A cutoff value of 2.0 was based on previously reported PIR value distribution among NHANES III population [11]. Of note, a PIR $<2.0$ corresponds to a low socioeconomic status. Family income was reported during the household interview. The poverty thresholds are adjusted for family size and are updated annually for inflation. BMI was calculated from the weight and height measured during the physical examination. Serum CRP was quantified using latex-enhanced nephelometry. Serum TC and HDL were measured enzymatically. Serum $25(\mathrm{OH}) \mathrm{D}$ concentrations were measured using a radioimmunoassay kit (DiaSorin Inc., Stillwater, Minn., USA) [12]. Serum creatinine was measured using the Jaffe modified kinetic method. The eGFR was calculated using the MDRD equation [13].

\section{Statistical Analysis}

We used SAS (9.3) PROC SURVEYMEANS and SURVEYFREQ to obtain descriptive statistics for the population. Characteristics of the population were compared between those with and without any history of kidney stone using the Rao-Scott $\chi^{2}$ for categorical variables and ANOVA for continuous variables. SAS (9.3) PROC SURVEYPHREG was used to perform Cox proportionalhazards regression analyses. Results were presented as a hazard ratio (HR) with 95\% CI. In Cox regression, we first examined the unadjusted HR of all-cause mortality and CV mortality. Then for model 1 , we added age (continuous variable), gender (male, female), race/ethnicity (NHW, non-Hispanic Black, Mexican American, and other), and PIR ( $<2.0$ vs. $\geq 2.0$ ). For model 2 , we added BMI (continuous variable), history of diabetes (yes, no), history of hypertension (yes, no), history of CV disease (yes, no), TC (continuous variable), HDL (continuous variable), and physical activity (continuous variable) in addition to those in model 1. For model 3, we added additional variables including eGFR (continuous variable), CRP (continuous variable), and serum 25(OH)D concentration (continuous variable) to model 2. Due to the complex sample strategy of NHANES III, appropriate 6-year weights and strata were applied. Because SAS was unable to generate surveyadjusted Kaplan-Meier plots, median follow-up times, and separate models for males and females, $\mathrm{R}$ version 2.15 .0 and its associated Survey package were used for these analyses. Previous analyses conducted in SAS were duplicated in $\mathrm{R}$ to confirm that all results were comparable. 
Table 1. Baseline characteristics of the study population

\begin{tabular}{lccc}
\hline & $\begin{array}{l}\text { Stone formers } \\
(\mathrm{n}=683)\end{array}$ & $\begin{array}{l}\text { Nonstone formers } \\
(\mathrm{n}=14,196)\end{array}$ & $\mathrm{p}$ \\
\hline Age, years & $54 \pm 0.8$ & $44 \pm 0.2$ & $<0.0001$ \\
Men & $419(61)$ & $6,558(46)$ & $<0.0001$ \\
NHW & $479(70)$ & $5,983(42)$ & $<0.0001$ \\
PIR $<2$ & $244(36)$ & $6,617(47)$ & $<0.0001$ \\
Hypertension & $275(40)$ & $3,712(26)$ & $<0.0001$ \\
Diabetes & $80(12)$ & $1,094(8)$ & 0.0001 \\
CV disease & $120(18)$ & $1,063(8)$ & $<0.0001$ \\
BMI & $27.8 \pm 0.3$ & $26.4 \pm 0.1$ & $<0.0001$ \\
CRP, mg/dl & $0.48 \pm 0.03$ & $0.41 \pm 0.01$ & 0.04 \\
25(OH)D, ng/ml & $29.6 \pm 0.6$ & $30.0 \pm 0.1$ & 0.5 \\
eGFR, ml/min/1.73 m ${ }^{2}$ & $63.5 \pm 0.8$ & $69.8 \pm 0.2$ & $<0.0001$ \\
TC, mg/dl & $213.7 \pm 2.1$ & $202.9 \pm 0.6$ & $<0.0001$ \\
HDL cholesterol, mg/dl & $47.6 \pm 0.8$ & $51.0 \pm 0.2$ & $<0.0001$ \\
Physical activity & $97.2 \pm 5.8$ & $114.3 \pm 1.8$ & 0.005 \\
\hline
\end{tabular}

Data presented as means \pm SE or $n$ (weighted $\%)$.

Table 2. Association of kidney stone disease with all-cause and CV mortality

\begin{tabular}{|c|c|c|c|c|c|c|}
\hline \multirow[t]{2}{*}{ Regression model } & \multicolumn{2}{|l|}{ Men } & \multicolumn{2}{|l|}{ Women } & \multicolumn{2}{|l|}{ Combined } \\
\hline & HR (95\% CI) & $\mathrm{p}$ & $\operatorname{HR}(95 \% \mathrm{CI})$ & $\mathrm{p}$ & HR (95\% CI) & $\mathrm{p}$ \\
\hline \multicolumn{7}{|l|}{ All-cause mortality } \\
\hline Unadjusted & $2.03(1.62-2.53)$ & $<0.0001$ & $1.81(1.35-2.42)$ & $<0.0001$ & $1.95(1.64-2.33)$ & $<0.0001$ \\
\hline Model 1 & $1.03(0.85-1.25)$ & 0.8 & $1.21(0.95-1.55)$ & 0.1 & $1.08(0.93-1.26)$ & 0.3 \\
\hline Model 2 & $0.99(0.81-1.20)$ & 0.9 & $1.05(0.82-1.34)$ & 0.7 & $1.00(0.85-1.17)$ & 1.0 \\
\hline Model 3 & $0.96(0.79-1.18)$ & 0.7 & $1.03(0.80-1.31)$ & 1.0 & $0.99(0.85-1.16)$ & 0.9 \\
\hline \multicolumn{7}{|l|}{ CV mortality } \\
\hline Unadjusted & $2.05(1.60-2.62)$ & $<0.0001$ & $2.06(1.40-3.04)$ & 0.0002 & $2.05(1.60-2.62)$ & $<0.0001$ \\
\hline Model 1 & $0.96(0.70-1.32)$ & 0.8 & $1.34(0.94-1.90)$ & 0.1 & $1.07(0.84-1.36)$ & 0.6 \\
\hline Model 2 & $0.88(0.64-1.22)$ & 0.5 & $1.09(0.79-1.51)$ & 0.6 & $0.94(0.74-1.19)$ & 0.6 \\
\hline Model 3 & $0.88(0.64-1.21)$ & 0.4 & $1.08(0.78-1.48)$ & 0.7 & $0.94(0.75-1.19)$ & 0.6 \\
\hline
\end{tabular}

Model 1 adjusted for age, gender, race, and PIR. Model 2 adjusted for BMI, TC, HDL, physical activity, history of hypertension, diabetes, and CV disease, in addition to the variables adjusted for in model 1. Model 3 adjusted for serum CRP, serum 25(OH)D, and $\mathrm{eGFR}$, in addition to the variables adjusted for in model 2.

\section{Results}

Out of 14,879 participants eligible for the final analysis, 683 reported a history of kidney stones. There were a total of 3,590 all-cause and 1,608 CV deaths during a median follow-up of 14.9 years. As shown in table 1, stone formers tended to be older, male, and NHW compared to nonstone formers. They also had a higher BMI, TC, and a lower HDL, and were more likely to have a history of hypertension, diabetes, and CV disease. In addition, stone formers had a higher serum CRP concentration and lower kidney function as measured by eGFR. Serum 25(OH)D concentrations were not different between stone formers and nonstone formers. Of note, $91 \%$ of the subjects in this study reported their income, and stone formers were more likely to have a higher socioeconomic status based on income.

Table 2 shows that kidney stone formers had a significantly higher risk of all-cause mortality (HR: 1.95, 95\% CI: 1.64-2.33, $\mathrm{p}<0.0001$ ) and CV mortality (HR: 2.05, 95\% CI: $1.60-2.62, \mathrm{p}<0.0001$ ) in unadjusted analysis (fig. 1,2 ). 


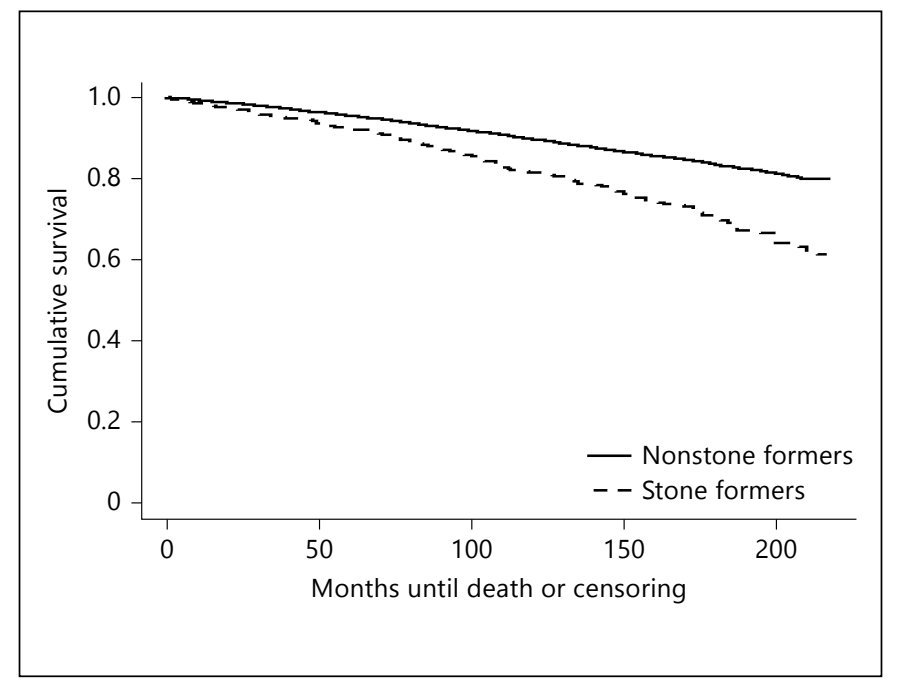

Fig. 1. Kaplan-Meier estimates for all-cause mortality (unadjusted). $\mathrm{p}<0.0001$.

After adjustment for age, gender, race, and PIR in model 1, stone formers no longer associated with increased allcause mortality (HR: $1.08,95 \%$ CI: $0.93-1.26, \mathrm{p}=0.3$ ) and CV mortality (HR: $1.07,95 \%$ CI: $0.84-1.36, \mathrm{p}=0.6$ ) in comparison to nonstone formers. After adding additional confounding variables in model 2 , including physical activity, history of hypertension, diabetes, CV disease, BMI, TC, and HDL, kidney stone disease was again not associated with increased hazard for all-cause mortality (HR: 1.00, 95\% CI: $0.85-1.17, \mathrm{p}=1.0$ ) or CV mortality (HR: $0.94,95 \%$ CI: $0.74-1.19, \mathrm{p}=0.6)$. Finally, there were no significant differences in all-cause mortality (HR: 0.99, 95\% CI: $0.85-1.16, \mathrm{p}=0.9$ ) and CV mortality (HR: 0.94, 95\% CI: $0.75-1.19, \mathrm{p}=0.6$ ) between stone formers and nonstone formers when eGFR, serum CRP, and 25(OH)D concentration were added to the final model (model 3 ). The stratified analyses by gender revealed similar findings.

Among the demographic variables in the analyses, age was found to be an important modifier of the mortality risk in this study. To confirm this age effect, we added age to the unadjusted model between kidney stone disease and allcause mortality, and found that kidney stone disease was nolonger associated with a higher risk of all-cause mortality (HR: $1.15,95 \%$ CI: 0.99-1.33, $\mathrm{p}=0.07$ ). As expected, age was a significant predictor of all-cause mortality in unadjusted analysis (HR per 10-year age increase: 2.40 , 95\% CI: $2.31-2.49, \mathrm{p}<0.0001)$. The additions of neither gender nor race alone into the unadjusted model resulted in any significant change in the association between kidney stone disease and all-cause mortality (data not shown).

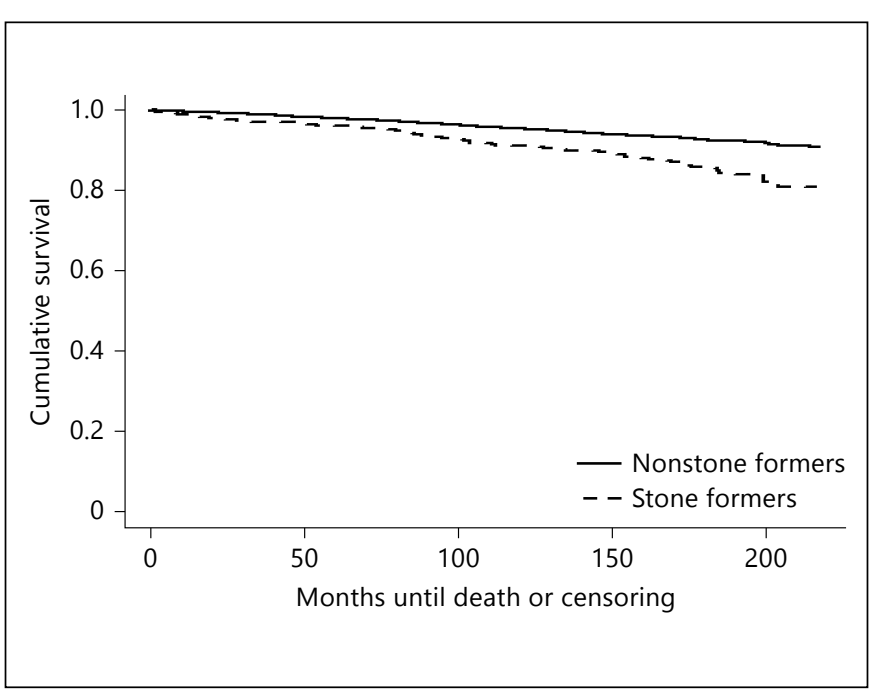

Fig. 2. Kaplan-Meier estimates for CV mortality (unadjusted). $\mathrm{p}<0.0001$.

In our multivariate Cox proportional hazard regression analyses (table 3 ), the following variables were found to have significant associations with all-cause mortality: older age (HR per 10-year age increase: $2.23,95 \%$ CI: 2.22-2.24, $\mathrm{p}<0.0001$ ), male gender (HR: 1.69, 95\% CI: 1.53-1.88, $\mathrm{p}<$ 0.0001 ), PIR <2 (HR: 1.53, 95\% CI: 1.37-1.71, p < 0.0001), history of hypertension (HR: 1.29, 95\% CI: 1.16-1.42, p < 0.0001 ), history of diabetes (HR: $1.68,95 \%$ CI: $1.47-1.92$, $\mathrm{p}<0.0001$ ), history of CV disease (HR: 1.65, 95\% CI: $1.47-$ $1.85, \mathrm{p}<0.0001$ ), and higher serum CRP (HR per 1-mg/dl increase: $1.20,95 \% \mathrm{CI}: 1.15-1.26, \mathrm{p}<0.0001)$. Of note, higher physical activity (HR per 1 -unit increase: $0.99,95 \% \mathrm{CI}$ : $0.99-0.99, \mathrm{p}=0.008$ ), increasing BMI (HR per $1-\mathrm{kg} / \mathrm{m}^{2}$ increase: $0.98,95 \%$ CI: $0.97-0.99, p=0.002$ ), and higher serum $25(\mathrm{OH}) \mathrm{D}$ concentrations (HR per $1-\mathrm{ng} / \mathrm{ml}$ increase: $0.99,95 \%$ CI: $0.98-0.99, \mathrm{p}=0.0003$ ) were associated with decreased all-cause mortality. The following variables were also strong predictors of CV mortality: older age (HR per 10-year age increase: $2.55,95 \%$ CI: $2.53-2.57, \mathrm{p}<0.0001$ ), male gender (HR: 1.86, 95\% CI: 1.58-2.18, $\mathrm{p}<0.0001$ ), PIR $<2$ (HR: 1.31, 95\% CI: 1.11-1.55, $\mathrm{p}=0.001$ ), history of hypertension (HR: 1.52, 95\% CI: 1.31-1.76, p <0.0001), history of diabetes (HR: 1.66, 95\% CI: 1.37-2.01, p < 0.0001), history of CV disease (HR: 2.28, 95\% CI: 1.95-2.67, p < 0.0001 ), higher TC (HR per 10-mg/dl increase: $1.03,95 \%$ CI: 1.03-1.03, $\mathrm{p}=0.0003$ ), and higher serum CRP (HR per $1-\mathrm{mg} / \mathrm{dl}$ increase: $1.18,95 \% \mathrm{CI}: 1.11-1.26, \mathrm{p}<0.0001)$. Increasing BMI (HR per 1-kg/ $\mathrm{m}^{2}$ increase: 0.98, 95\% CI: $0.97-$ $0.99, \mathrm{p}=0.04$ ), higher physical activity (HR per 1-unit increase: $0.99,95 \%$ CI: $0.99-0.99, p=0.02$ ), higher serum 
Table 3. Associations of demographics and other clinical variables with all-cause and CV mortality

\begin{tabular}{|c|c|c|c|c|}
\hline & \multicolumn{2}{|c|}{ All-cause mortality } & \multicolumn{2}{|l|}{ CV mortality } \\
\hline & $\mathrm{HR}(95 \% \mathrm{CI})$ & $\mathrm{p}$ & HR (95\% CI) & $\mathrm{p}$ \\
\hline Kidney stone & $0.99(0.85-1.16)$ & 0.9 & $0.94(0.75-1.19)$ & 0.6 \\
\hline Age (per 10-year increase) & $2.23(2.22-2.24)$ & $<0.0001$ & $2.55(2.53-2.57)$ & $<0.0001$ \\
\hline Men & $1.69(1.53-1.88)$ & $<0.0001$ & $1.86(1.58-2.18)$ & $<0.0001$ \\
\hline NHW & $0.97(0.85-1.10)$ & 0.6 & $0.99(0.82-1.19)$ & 0.9 \\
\hline Poverty income ratio $<2$ & $1.53(1.37-1.71)$ & $<0.0001$ & $1.31(1.11-1.55)$ & 0.001 \\
\hline Hypertension & $1.29(1.16-1.42)$ & $<0.0001$ & $1.52(1.31-1.76)$ & $<0.0001$ \\
\hline Diabetes & $1.68(1.47-1.92)$ & $<0.0001$ & $1.66(1.37-2.01)$ & $<0.0001$ \\
\hline CV disease & $1.65(1.47-1.85)$ & $<0.0001$ & $2.28(1.95-2.67)$ & $<0.0001$ \\
\hline BMI (per unit increase) & $0.98(0.97-0.99)$ & 0.002 & $0.98(0.97-0.99)$ & 0.04 \\
\hline Physical activity (per unit increase) & $0.99(0.99-0.99)$ & 0.008 & $0.99(0.99-0.99)$ & 0.02 \\
\hline TC (per 10-unit increase) & $1.00(0.99-1.00)$ & 0.9 & $1.03(1.03-1.03)$ & 0.0003 \\
\hline HDL (per unit increase) & $1.00(0.99-1.00)$ & 0.8 & $1.00(0.99-1.00)$ & 0.4 \\
\hline CRP (per unit increase) & $1.20(1.15-1.26)$ & $<0.0001$ & $1.18(1.11-1.26)$ & $<0.0001$ \\
\hline 25(OH)D (per unit increase) & $0.99(0.98-0.99)$ & 0.0003 & $0.99(0.98-0.99)$ & 0.002 \\
\hline eGFR (per 5-unit increase) & $0.99(0.98-1.00)$ & 0.2 & $0.96(0.95-0.96)$ & 0.01 \\
\hline
\end{tabular}

25(OH)D concentration (HR per $1-\mathrm{ng} / \mathrm{ml}$ increase: 0.99 , 95\% CI: 0.98-0.99, $\mathrm{p}=0.002$ ), and a higher eGFR (HR per $5-\mathrm{ml} / \mathrm{min} / 1.73 \mathrm{~m}^{2}$ increase: $0.96,95 \% \mathrm{CI}: 0.95-0.96, \mathrm{p}=$ $0.01)$ were associated with decreased CV mortality.

\section{Discussion}

To our knowledge, this is the first study examining the independent association of prevalent kidney stone disease with all-cause and CV mortality in the US adult population. We showed a significant increase in all-cause and CV mortality risk among stone formers in unadjusted analysis. However, after adjusting for age, other demographic variables, and vascular risk factors, the association of kidney stone disease with all-cause or CV mortality was no longer significant. This complete attenuation of the association suggests that the mortality risk among stone formers is likely a reflection of unique demographics and shared risk factors for mortality and kidney stone disease.

Consistent with findings from earlier studies $[1,5,14-$ 16], stone formers in our study were older and had a male predominance. They were also more likely to have hypertension, diabetes, and obesity. Hypertension among stone formers has been linked to a reduction of urinary citrate excretion, and thus promotes stone formation [17]. Both glucose intake and insulin administration can reduce calcium reabsorption [18-20], suggesting that a high glucose and insulin state from insulin-resistance in diabetes and obesity can increase urinary calcium excretion. In addi- tion, by suppressing renal tubular ammonium production [21], insulin resistance can lead to more acidified urine and increase the risk of uric acid stone formation [22]. Prevalent chronic kidney disease (CKD) was also more common among stone formers in our study population, consistent with what has been reported by Gillen et al. [23] and other investigators [9,24]. It is unclear why stone formers develop CKD, as evidence suggests CKD risk among stone formers is independent of traditional risk factors such as hypertension and diabetes [9]. Stone type (in particular, cystine and struvite stones, among others) could be the contributing factor for developing CKD, although potential mechanisms are not clear [25].

Because of the strong associations of the above-mentioned CV disease risk factors with prevalent kidney stone disease, it is not surprising to see a significantly increased risk of $\mathrm{CV}$ disease among stone formers in our study population. This is consistent with the findings reported previously by Reiner et al. [6] who showed a strong association between kidney stone disease and carotid wall thickness among CARDIA study participants. Rule et al. [7] compared 4,564 kidney stone formers to 10,860 matched controls from Olmsted County (Minn., USA), and reported a $31 \%$ increased risk for new-onset myocardial infarction in stone formers after adjustment for CKD and other comorbidities.

Among all the demographic and clinical variables included in this study, older age, male gender, poverty, physical inactivity, history of hypertension, diabetes, CV disease, reduced body vitamin $\mathrm{D}$ store, and inflammatory 
state were strong predictors of all-cause mortality and CV mortality. These are consistent with the mortality risk factors previously described in the general population [26-28]. Reduced kidney function was also noted to be a predictor for CV mortality, as it has been reported in the US adult population [29]. Furthermore, higher BMI appeared to have a mild protective effect on both all-cause mortality and $\mathrm{CV}$ mortality. This was unexpected considering the high mortality risk among the overweight general population [30].

Limitations of our study need to be mentioned. First, the prevalent kidney stone cases were self-reported, and some participants may have kidney stone disease without self-awareness or clinical diagnosis. This may lead to potential misclassification and is likely to be random with respect to case status. Therefore, it could bias the study results toward thenull. Second, we donothaveinformation on stone composition, although approximately $80 \%$ of kidney stones in the general population, like NHANES, are calcium based. It is unclear whether patients with a different type of kidney stone carry different mortality risks. Lastly, due to the observational study design and the nature of the NHANES survey database, we cannot establish any causal relationships.

In summary, the increased risk of all-cause and CV mortality among kidney stone formers is likely a reflection of unique demographics and associated comorbidities. There is no independent association of prevalent kidney stone disease with mortality. Future studies in other populations are needed to confirm these results.

\section{Acknowledgement}

This manuscript is supported in part by NIH/NIDDK grants 1R01DK094796 and 1R01DK081473-01. All authors declare that they have no relevant financial interests.

\section{References}

$\checkmark 1$ Stamatelou KK, et al: Time trends in reported prevalence of kidney stones in the United States: 1976-1994. Kidney Int 2003;63:18171823.

2 Scales CD Jr, et al: Prevalence of kidney stones in the United States. Eur Urol 2012;62:160165.

-3 Saigal CS, Joyce G, Timilsina AR: Direct and indirect costs of nephrolithiasis in an employed population: opportunity for disease management? Kidney Int 2005;68:1808-1814.

-4 Taylor EN, Stampfer MJ, Curhan GC: Obesity, weight gain, and the risk of kidney stones. JAMA 2005;293:455-462.

5 Taylor EN, Stampfer MJ, Curhan GC: Diabetes mellitus and the risk of nephrolithiasis. Kidney Int 2005;68:1230-1235.

6 Reiner AP, et al: Kidney stones and subclinical atherosclerosis in young adults: the CAR DIA study. J Urol 2011;185:920-925.

7 Rule AD, et al: Kidney stones associate with increased risk for myocardial infarction. J Am Soc Nephrol 2010;21:1641-1644.

8 Alexander RT, et al: Kidney stones and kidney function loss: a cohort study. BMJ 2012; 345:e5287.

9 Rule $\mathrm{AD}$, et al: Kidney stones and the risk for chronic kidney disease. Clin J Am Soc Nephrol 2009;4:804-811.

10 The Third National Health and Nutrition Examination Survey (NHANES III) Linked Mortality File, Mortality Follow-Up through 2006: Matching Methodology. 2009. http:// www.cdc.gov/nchs/data/datalinkage/ matching_methodology_nhanes3_final.pdf.

-11 Sabanayagam C, Shankar A: Income is a stronger predictor of mortality than education in a national sample of US adults. J Health Popul Nutr 2012;30:82-86.

12 The Third National Health and Nutrition Examination Survey (NHANES III 1988-94) Reference Manuals and Reports (book on CD-ROM). Bethesda, National Center for Health Statistics, 2002.

13 Stevens LA, et al: Evaluation of the Modification of Diet in Renal Disease study equation in a large diverse population. J Am Soc Nephrol 2007;18:2749-2757.

14 Cirillo M, Laurenzi M: Elevated blood pressure and positive history of kidney stones: results from a population-based study. J Hypertens Suppl 1988;6:S485-S486.

15 Cappuccio FP, et al: A prospective study of hypertension and the incidence of kidney stones in men. J Hypertens 1999;17:1017-1022.

16 Madore F, et al: Nephrolithiasis and risk of hypertension in women. Am J Kidney Dis 1998:32:802-807.

17 Taylor EN, et al: Association of prevalent hypertension with 24-hour urinary excretion of calcium, citrate, and other factors. Am J Kidney Dis 2006;47:780-789.

18 Lemann J Jr, et al: Evidence that glucose ingestion inhibits net renal tubular reabsorption of calcium and magnesium in man. J Lab Clin Med 1970;75:578-585.

19 Lemann J Jr, Piering WF, Lennon EJ: Possible role of carbohydrate-induced calciuria in calcium oxalate kidney-stone formation. $\mathrm{N}$ Engl J Med 1969;280:232-237.

20 DeFronzo RA, et al: The effect of insulin on renal handling of sodium, potassium, calcium, and phosphate in man. J Clin Invest 1975;55: 845-855.
21 Chobanian MC, Hammerman MR: Insulin stimulates ammoniagenesis in canine renal proximal tubular segments. Am J Physiol 1987;253:F1171-F1177.

22 Asplin JR: Uric acid stones. Semin Nephrol 1996;16:412-424.

23 Gillen DL, Worcester EM, Coe FL: Decreased renal function among adults with a history of nephrolithiasis: a study of NHANES III. Kidney Int 2005;67:685-690.

24 Vupputuri S, et al: History of kidney stones as a possible risk factor for chronic kidney disease. Ann Epidemiol 2004;14:222-228.

25 Worcester EM, et al: Renal function in patients with nephrolithiasis. J Urol 2006;176: 600-603, discussion 603.

26 Lee IM, et al: Effect of physical inactivity on majornon-communicablediseases worldwide: an analysis of burden of disease and life expectancy. Lancet 2012;380:219-229.

-27 Emerging Risk Factors Collaboration, Seshasai SR, Kaptoge S, Thompson A, et al: Diabetes mellitus, fasting glucose, and risk of cause-specific death. N Engl J Med 2011;364:829-841.

28 Ford ES: Trends in mortality from all causes and cardiovascular disease among hypertensive and nonhypertensive adults in the United States. Circulation 2011;123:1737-1744.

29 Muntner P, et al: Renal insufficiency and subsequent death resulting from cardiovascular disease in the United States. J Am Soc Nephrol 2002;13:745-753.

$30 \mathrm{Hu}$ G, et al: Prevalence of the metabolic syndrome and its relation to all-cause and cardiovascular mortality in nondiabetic European men and women. Arch Intern Med 2004;164: 1066-1076. 